\title{
Head-on collision of internal waves with trapped cores
}

\author{
Vladimir Maderich ${ }^{1}$, Kyung Tae Jung ${ }^{2}$, Kateryna Terletska ${ }^{1}$, and Kyeong Ok Kim ${ }^{2}$ \\ ${ }^{1}$ Institute of Mathematical Machine and System Problems, Glushkov av., 42, Kiev 03187, Ukraine \\ ${ }^{2}$ Korea Institute of Ocean Science and Technology, 787, Haean-ro, Ansan 426-744 Republic of Korea
}

Correspondence: Vladimir Maderich (vladmad@gmail.com)

Received: 1 July 2017 - Revised: 4 November 2017 - Accepted: 15 November 2017 - Published: 22 December 2017

\begin{abstract}
The dynamics and energetics of a head-on collision of internal solitary waves (ISWs) with trapped cores propagating in a thin pycnocline were studied numerically within the framework of the Navier-Stokes equations for a stratified fluid. The peculiarity of this collision is that it involves trapped masses of a fluid. The interaction of ISWs differs for three classes of ISWs: (i) weakly non-linear waves without trapped cores, (ii) stable strongly non-linear waves with trapped cores, and (iii) shear unstable strongly nonlinear waves. The wave phase shift of the colliding waves with equal amplitude grows as the amplitudes increase for colliding waves of classes (i) and (ii) and remains almost constant for those of class (iii). The excess of the maximum run-up amplitude, normalized by the amplitude of the waves, over the sum of the amplitudes of the equal colliding waves increases almost linearly with increasing amplitude of the interacting waves belonging to classes (i) and (ii); however, it decreases somewhat for those of class (iii). The colliding waves of class (ii) lose fluid trapped by the wave cores when amplitudes normalized by the thickness of the pycnocline are in the range of approximately between 1 and 1.75. The interacting stable waves of higher amplitude capture cores and carry trapped fluid in opposite directions with little mass loss. The collision of locally shear unstable waves of class (iii) is accompanied by the development of instability. The dependence of loss of energy on the wave amplitude is not monotonic. Initially, the energy loss due to the interaction increases as the wave amplitude increases. Then, the energy losses reach a maximum due to the loss of potential energy of the cores upon collision and then start to decrease. With further amplitude growth, collision is accompanied by the development of instability and an increase in the loss of energy. The collision process is modified for waves of different amplitudes because of the exchange of trapped fluid
\end{abstract}

between colliding waves due to the conservation of momentum.

\section{Introduction}

Internal solitary waves (ISWs) are widespread in stratified oceans and lakes (Helfrich and Melville, 2006). The observed ISWs are mostly waves of mode-1 and propagate as waves of depression or as waves of elevation. When nearsurface or near-bottom layers are stratified, then mode-1 ISWs of large amplitude can trap and transport fluid in their cores, as observed in the ocean (Moum et al., 2003; Lien et al., 2012; Klymak and Moum, 2003; Scotti and Pineda, 2004) and in the atmospheric boundary layer (the phenomenon known as "the morning glory"; Christie, 1992; Reeder et al., 1995). These waves have been studied in laboratory experiments (Grue et al., 2000; Carr et al., 2008; Luzzatto-Fegiz and Helfrich, 2014). The fluid can also be trapped by ISWs of mode-2 (Yang et al., 2010; Shroyer et al., 2010; Ramp et al., 2015) propagating in the thin interface layer between two uniform density layers, as has been shown in laboratory experiments (e.g. Davis and Acrivos, 1967; Maxworthy, 1980; Kao and Pao, 1980; Honji and Matsunaga, 1995; Stamp and Jacka, 1995; Maderich et al., 2001; Brandt and Shipley, 2014; Carr et al., 2015). The weakly non-linear solution for the corresponding ISW (i.e. the Benjamin-Ono (BO) soliton; Benjamin, 1967; Ono, 1975) agrees well with experimental data for a small-amplitude ISW without mass transport. However, experiments and numerical solutions of both the Dubreil-Jacotin-Long (DJL) equation and the actual Navier-Stokes equations (Lamb, 2002; Helfrich and White, 2010; Lamb and Farmer, 2011; Salloum et al., 2012; Maderich et al., 2015; Luzzatto-Fegiz and Helfrich, 2014; Deepwell and Stastna, 2016) show that BO solitons cannot 
even qualitatively describe the dynamics and transport features of large-amplitude waves. A detailed review of laboratory and numerical experiments is given by Maderich et al. (2015).

Little is known regarding the interaction of waves with trapped cores. This kind of interaction is of special interest, as the masses of fluid trapped by waves are also involved in the interaction. The oblique interaction of "morning glories" over northern Australia was described by Reeder et al. (1995). Head-on collision as a special case of oblique interaction was considered by Matsuno (1998) using a second-order analysis of small-amplitude interfacial waves in deep fluid. A mostly qualitative description of the head-on collision of mode- 2 waves with trapped cores, obtained by conducting several laboratory experiments (Kao and Pao, 1980; Honji and Matsunaga, 1995; Stamp and Jacka, 1995; Gavrilov et al., 2012) and via a numerical simulation (Terez and Knio, 1998), has been given. These experiments showed that (i) waves experience a phase shift during collision, (ii) largeamplitude waves transport trapped masses of fluid after collision, (iii) waves of unequal amplitude exchange masses of trapped fluid in the interaction process, and (iv) some trapped fluid is ejected upon collision. According to Gear and Grimshaw (1984), interaction processes can be distinguished as strong interactions when waves propagate in almost the same direction and the time of interaction is relatively long and as weak interaction when waves propagate in almost opposite directions and the time of interaction is relatively short. However, a numerical study of both overtaking and head-on collisions of large-amplitude mode- 1 and weakly non-linear mode-2 ISWs Stastna et al. (2015) showed that these interactions are strong interactions resulting in the degeneration of mode-2 ISWs. In this paper, the dynamics and energetics of a head-on collision of ISWs with trapped cores for a wide range of amplitudes and stratifications are studied numerically within the framework of the Navier-Stokes equations. The paper is organized as follows: the numerical flume set-up is described in Sect. 2, the results of experiments on the collision of waves of equal and non-equal amplitudes are discussed in Sect. 3, and the main results are summarized in Sect. 4.

\section{The numerical model set-up}

A free-surface non-hydrostatic numerical model for variabledensity flows using the Navier-Stokes equations under the Boussinesq approximation (Kanarska and Maderich, 2003; Maderich et al., 2012) was applied in the simulations of a numerical flume emulating a laboratory basin filled with salinity-stratified water. The numerical flume and experimental configurations are shown in Fig. 1. The simulations were performed in a 2-D setting where $(x, y)$ are the Cartesian coordinates in the longitudinal and vertical directions, respectively. The vertical coordinate $z$ is directed upward. The

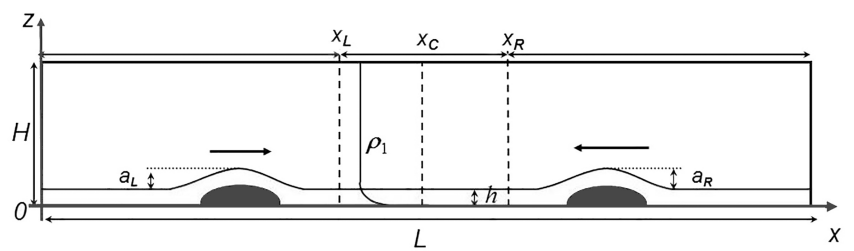

Figure 1. Configuration of the experiment exploring the interaction of ISWs with trapped cores.

flume has a length $L_{x}=3 \mathrm{~m}$ and a depth $H=0.46 \mathrm{~m}$. It was filled with salinity $S$ stratified water, in which the density of the upper layer is $\rho_{1}$, and with a thin pycnocline near the bottom, expressed as

$\rho(z)=\rho_{0}\left(1-\frac{\Delta \rho}{\rho_{0}} \tanh \left(\frac{z}{h}\right)\right)$,

where $\rho_{0}$ is the density at the bottom, $\Delta \rho=\rho_{0}-\rho_{1}$ is the density difference between the bottom and the surface, and $h$ is a parameter of the pycnocline. An equation of state $\rho=$ $\rho(S, T)$ (Mellor, 1991) was used for constant temperature $T=15^{\circ} \mathrm{C}$.

The ISWs were generated at both ends of the flume by the collapse of the mixed regions with the density $\rho_{0}$. Following Maderich et al. (2015), the shape of the mixed region was selected to be half of a $\mathrm{BO}$ soliton to reduce the mixing due to the collapse. The wave amplitude varied according to the initial volume of the mixed fluid and thickness of the pycnocline $h$. The kinematic viscosity $v$ was $1.14 \times 10^{-6} \mathrm{~m}^{2} \mathrm{~s}^{-1}$, and the molecular diffusion $\chi$ was $10^{-9} \mathrm{~m}^{2} \mathrm{~s}^{-1}$.

An ISW is characterized by an amplitude $a$, which represents the maximum displacement of the isopycnal (Fig. 1), a wave speed $U_{\mathrm{c}}$, calculated as the velocity of the wave crest, and a wavelength $\lambda_{0.5}$, estimated to be a half-width with which the amplitude of the wave is reduced by half. The maximal speed of the wave is $U_{\mathrm{m}}$. The wave parameters were evaluated in the sections $x_{\mathrm{L}}=x_{\mathrm{R}}=0.5 \mathrm{~m}$ away from the centre of the laboratory tank $x_{\mathrm{C}}$. For example, the amplitudes of waves propagating from the left to the right in cross sections $x_{\mathrm{L}}$ and $x_{\mathrm{R}}$ are defined as $a_{\mathrm{L}}\left(x_{\mathrm{L}}\right)$ and $a_{\mathrm{L}}\left(x_{\mathrm{R}}\right)$, while those propagating from right to left are defined as $a_{\mathrm{R}}\left(x_{\mathrm{L}}\right)$ and $a_{\mathrm{R}}\left(x_{\mathrm{R}}\right)$, respectively.

The simulation results are presented in dimensionless form. The coordinates $x_{i}=(x, z)$ are normalized by $h$, and the time $\tau=t / \tau_{0}$ is dimensionless, where $t$ is time, $\tau_{0}=$ $\sqrt{2 \rho_{0} h / \Delta \rho g}$, and $g$ is gravity; the velocity $u_{i}=(u, w)$ is normalized to the long linear wave phase velocity $C_{0}=$ $\sqrt{g h \Delta \rho / 2 \rho_{0}}$ (Benjamin, 1967); $p$ is pressure deviation in the Boussinesq approximation normalized to the $\rho_{0} g h / 2$; $\rho^{\prime}=\left(\rho-\rho_{0}\right) / \rho_{0}$ is non-dimensional density deviation. The governing Navier-Stokes equations for stratified fluid in nondimensional form are written under the Boussinesq approxi- 
mation as

$$
\begin{aligned}
\frac{\partial u_{i}}{\partial x_{i}}=0 & \\
\frac{\partial u_{i}}{\partial \tau}+u_{j} \frac{\partial u_{i}}{\partial x_{j}}= & -\frac{\partial p}{\partial x_{i}}-\frac{1}{2} \rho^{\prime} \delta_{i, 3} \\
& +\frac{1}{\operatorname{Re}} \frac{\partial}{\partial x_{j}}\left(\frac{\partial u_{i}}{\partial x_{j}}+\frac{\partial u_{j}}{\partial x_{i}}\right), \\
\frac{\partial \rho^{\prime}}{\partial \tau}+u_{j} \frac{\partial \rho^{\prime}}{\partial x_{j}}= & \frac{1}{\operatorname{Re} S c} \frac{\partial^{2} \rho^{\prime}}{\partial x_{j} \partial x_{j}},
\end{aligned}
$$

where $\delta_{i j}$ is the Kronecker delta, $R e=C_{0} h / v$ is the Reynolds number based on linear theory, and $S c=v / \chi$ is the Schmidt number. The effect of the height of the computational tank on the ISW propagation is described by the parameter $\varepsilon=H / h$. It can be assumed that the results of experiments and simulation for small viscosity $(\operatorname{Re} \rightarrow \infty)$, small diffusivity for water $(S c \rightarrow \infty)$, and deep water $(\varepsilon \rightarrow \infty)$ will not depend on the viscosity, diffusivity, and depth. That case is referred to by Barenblatt (1996) as a complete similarity in the parameters $R e, S c$, and $\varepsilon$. Generally, however, the flow dependence on the viscosity, diffusivity, and depth can be retained at $R e \rightarrow \infty, S c \rightarrow \infty$, and $\epsilon \rightarrow \infty$, and scaling on them is called incomplete (Barenblatt, 1996). In most cases it is impossible to determine the kind of self-similarity a priori, until the solution of the full problem is available. Like (Maderich et al., 2015), we follow the suggestion by Barenblatt (1996) "assuming in succession complete similarity, incomplete similarity, lack of similarity - and then comparing the relations obtained under each assumption with data from numerical calculations, experiments, or the results of analytic investigations". The simulation results (Maderich et al., 2015) show that the flume depth in the range $23 \leq \varepsilon \leq 92$ does not affect the characteristics of the ISWs with trapped cores. The sensitivity of the wave dynamics to the values of $\varepsilon$ was found by Carr et al. (2008) in the range $4 \leq \varepsilon \leq 11$. From these studies we conclude that results of our simulations in the range $23 \leq \varepsilon \leq 92$ (Table 1) do not depend on $\varepsilon$. The possible effects of Schmidt and Reynolds numbers will be discussed in Sect. 3.4 and 3.6.

The important features of the ISWs can be described by the dimensionless amplitude $\alpha=a / h$, and the Froude, Richardson, and Reynolds numbers based on local characteristics of the waves (Maderich et al., 2015). The Froude number $F r_{\text {max }}$ is defined as the ratio of the maximum local velocity $U_{\mathrm{m}}$ to the phase velocity $U_{\mathrm{c}}$ :

$F r_{\text {max }}=\frac{U_{\mathrm{m}}}{U_{\mathrm{c}}}$.

The shear stability of an ISW can be described by the minimum Richardson number $R i_{\min }$ calculated for a wave crest:

$R i_{\min }=\frac{g}{\rho_{0}} \frac{\partial \rho}{\partial z} /\left(\frac{\partial U}{\partial z}\right)^{2}$, where $\rho(x, y, z, t)$ is the density, and $U$ is the longitudinal component of velocity. The ISW Reynolds number is defined as

$R e_{\mathrm{m}}=\frac{U_{\mathrm{m}}(a)}{v}$.

The parameters of the interacting ISWs studied are given in Table 1. The waves are divided into four groups: (A, B, C) depending on the thickness of the stratified layer and D for simulation of ISW reflection from a vertical wall in the laboratory experiment (Stamp and Jacka, 1995). The waves in Table 1 can be categorized according to the values of the parameters $F r_{\max }$ and $R i_{\min }$ (Maderich et al., 2015) into three classes: (i) the weakly non-linear waves without trapped cores at $1<R i_{\min }, F r_{\max }<1$; (ii) the stable strongly non-linear waves with trapped cores at $0.15<R i_{\min }<1,1<$ $F r_{\text {max }}<1.3$; and (iii) the unstable strongly non-linear waves with trapped cores at $R i_{\min }<0.1 ; F r_{\max } \approx 1.35$. The boundary conditions on the surface include the kinematic condition for the free surface. At the rest of the boundaries, the freeslip conditions are used. The no-flux condition for density deviation was applied at all boundaries. For large $\varepsilon$, these allow for the simulation of the interaction of mode-1 ISWs with a trapped core, propagating in stratified layers near the surface and the ISWs interaction near the bottom, as considered here, and the interaction of mode- 2 ISWs, assuming symmetry in the Boussinesq approximation around the horizontal midplane (Maderich et al., 2015). The numerics of the model is described in detail in (Kanarska and Maderich, 2003; Maderich et al., 2012). A total of 40 runs were performed in Series A-D. Most of the runs were performed with a grid resolution of $3000 \times 400$ (length and height, respectively), whereas several runs (waves A11-A13) were also carried out with a grid resolution of $6000 \times 800$ to verify effects of grid resolution on the wave interaction and to make the finest structure clearer. Comparison of the main and doubled grid resolution showed the equivalence of the calculated fields, with the exception of wave A13, for which $6000 \times 800$ resolution was used.

\section{Results and discussion}

\subsection{Interaction of waves of equal amplitudes without trapped cores}

The interaction of ISWs of equal amplitude $\alpha_{\mathrm{L}}=\alpha_{\mathrm{R}}=0.81$ (case (A2; A2)) is shown in Fig. 2a. These waves belong to the class of weakly non-linear waves without trapped cores $\left(F r_{\max }=0.71, R i_{\min }=52\right)$. After a collision, waves retain their profile and lose amplitude mainly due to viscous effects. Before and after collision, the wave profiles are similar to those of the weakly non-linear BO solitons even if the wave amplitudes are not small (Fig. 3). The collision for 
Table 1. Summary of parameters of interacting ISWs: pycnocline thickness parameter $h$, wave amplitude $a$, wavelength $\lambda_{0.5}$, ratio $\varepsilon$, dimensionless ISW amplitude $\alpha$, Froude number $F r_{\max }$, minimum Richardson number $R i_{\text {min }}$, Reynolds number $R e_{\mathrm{m}}$, and ISW class.

\begin{tabular}{|c|c|c|c|c|c|c|c|c|c|}
\hline Wave & $\begin{array}{r}h \\
(\mathrm{~cm})\end{array}$ & $\begin{array}{r}a \\
(\mathrm{~cm})\end{array}$ & $\begin{array}{l}\lambda_{0.5} \\
(\mathrm{~cm})\end{array}$ & $\varepsilon$ & $\alpha$ & $F r_{\max }$ & $R i_{\min }$ & $R e_{\mathrm{m}}$ & $\begin{array}{l}\text { Wave } \\
\text { class }\end{array}$ \\
\hline A1 & 0.5 & 0.25 & 3.15 & 92 & 0.5 & 0.33 & 81 & 45.1 & $\mathrm{i}$ \\
\hline $\mathrm{A} 2$ & 0.5 & 0.4 & 2.35 & 92 & 0.81 & 0.71 & 52 & 86.6 & $\mathrm{i}$ \\
\hline A3 & 0.5 & 0.58 & 1.9 & 92 & 1.15 & 0.82 & 14 & 132 & $\mathrm{i}$ \\
\hline A4 & 0.5 & 0.68 & 2 & 92 & 1.35 & 0.98 & 11 & 166 & i \\
\hline A5 & 0.5 & 0.8 & 2.2 & 92 & 1.6 & 1.11 & 1.1 & 223 & ii \\
\hline A6 & 0.5 & 0.94 & 2.4 & 92 & 1.88 & 1.12 & 0.8 & 277 & ii \\
\hline A7 & 0.5 & 1.08 & 2.65 & 92 & 2.15 & 1.16 & 0.4 & 338 & ii \\
\hline A8 & 0.5 & 1.3 & 3.15 & 92 & 2.6 & 1.25 & 0.35 & 443 & ii \\
\hline A9 & 0.5 & 1.7 & 3.65 & 92 & 3.3 & 1.28 & 0.25 & 683 & ii \\
\hline A10 & 0.5 & 1.9 & 4.25 & 92 & 3.38 & 1.29 & 0.19 & 785 & ii \\
\hline A11 & 0.5 & 2.3 & 4.75 & 92 & 4.6 & 1.3 & 0.15 & 1075 & ii \\
\hline A12 & 0.5 & 2.5 & 5.35 & 92 & 5 & 1.35 & 0.12 & 1242 & iii \\
\hline A13 & 0.5 & 3.2 & 6.35 & 92 & 6.4 & 1.31 & 0.06 & 1681 & iii \\
\hline B1 & 1 & 0.63 & 5.21 & 46 & 0.63 & 0.51 & 24 & 153 & $\mathrm{i}$ \\
\hline B2 & 1 & 0.85 & 4.23 & 46 & 0.85 & 0.68 & 11.5 & 225 & $\mathrm{i}$ \\
\hline B3 & 1 & 1.25 & 3.61 & 46 & 1.25 & 1.02 & 2.4 & 388 & ii \\
\hline B4 & 1 & 1.95 & 5.25 & 46 & 1.95 & 1.16 & 0.38 & 765 & ii \\
\hline B5 & 1 & 2.68 & 6.65 & 46 & 2.68 & 1.22 & 0.18 & 1225 & ii \\
\hline B6 & 1 & 2.86 & 7.1 & 46 & 2.86 & 1.22 & 0.13 & 1345 & ii \\
\hline B7 & 1 & 3.56 & 8.6 & 46 & 3.56 & 1.23 & 0.11 & 1839 & iii \\
\hline $\mathrm{C} 1$ & 2 & 0.42 & 14 & 23 & 0.21 & 0.19 & 52 & 161 & $\mathrm{i}$ \\
\hline $\mathrm{C} 2$ & 2 & 0.76 & 10.4 & 23 & 0.38 & 0.30 & 25 & 291 & $\mathrm{i}$ \\
\hline $\mathrm{C} 3$ & 2 & 1.2 & 7 & 23 & 0.6 & 0.50 & 3.1 & 511 & $\mathrm{i}$ \\
\hline $\mathrm{C} 4$ & 2 & 1.7 & 6.1 & 23 & 0.85 & 0.69 & 1.1 & 669 & $\mathrm{i}$ \\
\hline C5 & 2 & 2.02 & 6.21 & 23 & 1.01 & 0.84 & 0.45 & 881 & $\mathrm{i}$ \\
\hline C6 & 2 & 2.6 & 6.25 & 23 & 1.3 & 0.9 & 0.23 & 1159 & i \\
\hline C7 & 2 & 2.9 & 7.22 & 23 & 1.45 & 1.01 & 0.22 & 1483 & ii \\
\hline C8 & 2 & 3.3 & 7.9 & 23 & 1.65 & 1.08 & 0.18 & 1851 & ii \\
\hline C9 & 2 & 3.5 & 8.5 & 23 & 1.75 & 1.147 & 0.15 & 2030 & ii \\
\hline $\mathrm{C} 10$ & 2 & 4.1 & 9.2 & 23 & 2.05 & 1.17 & 0.13 & 2521 & ii \\
\hline $\mathrm{C} 11$ & 2 & 4.56 & 10.82 & 23 & 2.28 & 1.23 & 0.12 & 2812 & ii \\
\hline $\mathrm{C} 12$ & 2 & 4.846 & 12.44 & 23 & 2.42 & 1.24 & 0.09 & 3171 & iii \\
\hline C13 & 2 & 5.28 & 13.4 & 23 & 2.64 & 1.25 & 0.07 & 3478 & iii \\
\hline C14 & 2 & 5.94 & 15.31 & 23 & 2.97 & 1.29 & 0.05 & 3884 & iii \\
\hline D1 & 0.25 & 0.55 & 12.5 & 56 & 2.2 & 1.18 & 1.05 & 329 & ii \\
\hline
\end{tabular}

this class of wave is not fully elastic. For a two-layer stratification, in which the outer layer is assumed to be infinitely deep, a weakly non-linear theory (Matsuno, 1998) predicts excess $\Delta \alpha$ of the maximum runup $\alpha_{\mathrm{m}}$ over the sum of the amplitudes of equal interacting waves $\alpha$ as $\Delta \alpha \sim \alpha^{2}$. In contrast, normalized to characteristic time $\tau_{0}$, the temporal phase shift $\Delta \theta$ is $\Delta \theta \sim \alpha$. The presence of a phase shift due to the collision of mode- 2 waves for $\alpha=0.98$ was confirmed in a laboratory experiment (Honji and Matsunaga, 1995). We estimated the temporal phase shift by comparing trajectories of the wave crests with and without collision. As seen in Fig. 4, the relative runup excess $\Delta \alpha / \alpha$ and normalized temporal phase shift $\Delta \theta$ increase as the interacting wave amplitude $\alpha$ increases at small and moderate $\alpha$, as weakly non-linear theory predicts.

\subsection{Interaction of waves with a trapped core and moderate amplitude}

The head-on collision between ISWs of equal, moderate amplitude with trapped cores $\alpha=\alpha_{\mathrm{L}}=\alpha_{\mathrm{R}}=1.6$ (case (A5; A5)) is characterized by special features, as seen in Fig. $2 b$. These waves, belonging to class (ii), i.e. stable strongly nonlinear waves with trapped cores $\left(F r_{\max }=1.11, R i_{\min }=1.1\right)$, carried fluid in the cores before the collision. The trapped fluid slowly leaks from the rear of the trapped bulge, similarly to the laboratory experiments (e.g. Maderich et al., 
(a)
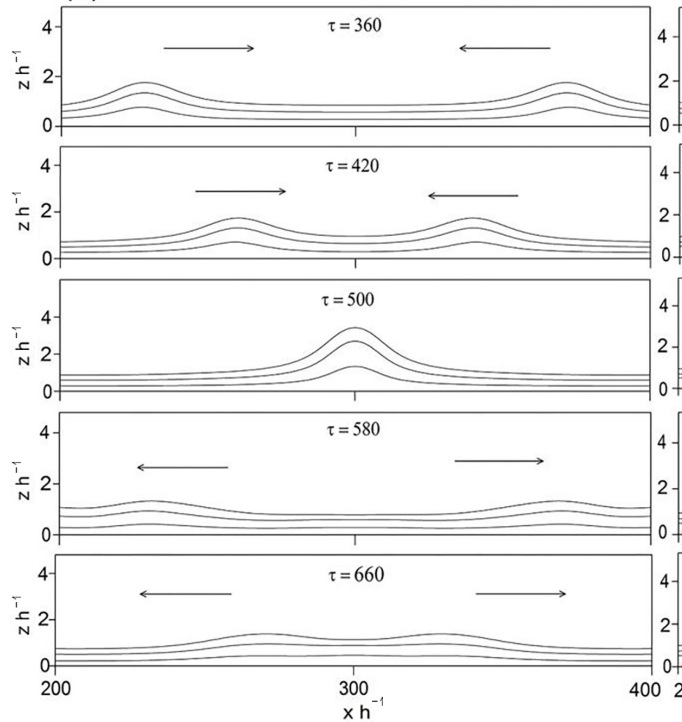

(b)
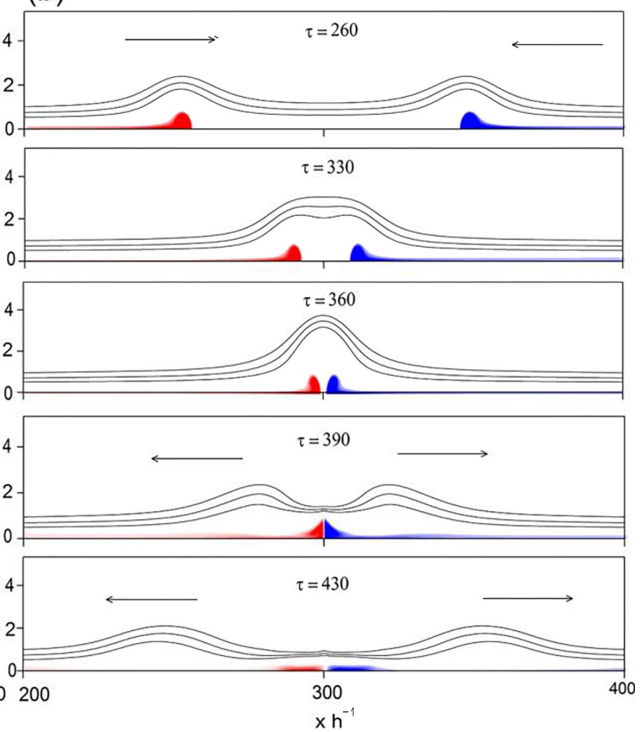

Figure 2. Snapshots of the density isopycnals during the collision of ISWs. (a) Case (A2; A2). (b) Case (A5; A5). The trapped cores are visualized by dyed fluid.
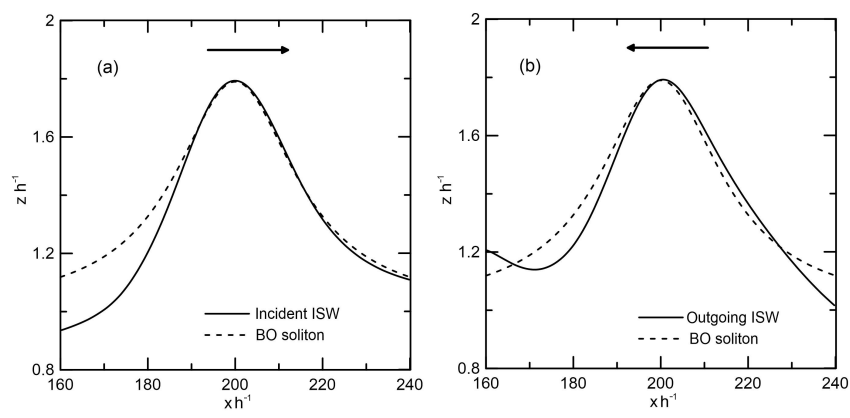

Figure 3. Wave profile of a wave before (a) and after (b) collision in section $x_{\mathrm{L}}$ (b) for $\alpha=0.81$ (case A2; A2). These profiles are compared with the profile of the $\mathrm{BO}$ soliton.

2001; Brandt and Shipley, 2014). However, after collision, the waves lost all fluid trapped by the wave cores. This fluid slowly collapsed in the viscous and diffusive-viscous regimes (Galaktionov et al., 2001). The profile of the incident wave at $\alpha=1.6$ (as well as other characteristics: Maderich et al., 2015) essentially differs from the predictions made by using the weakly non-linear theory (Fig. 5a). The amplitudes of interacting waves $\left(F r_{\max }=1.0, R i_{\min }=1.2\right)$ decrease after collision. They propagate as weakly non-linear BO solitons (Fig. 5b). This kind of head-on collision occurs in the range of approximately $1 \leq \alpha \leq 1.6$. Note that in the numerical study (Terez and Knio, 1998), the wave lost trapped fluid in the process of interaction even at $\alpha=2.1$. As shown in Fig. 4, the normalized excess of the maximum amplitude $\Delta \alpha$ increases almost linearly in the range $1 \leq \alpha \leq 2$, whereas the increase in the phase shift $\Delta \theta$ slows down.
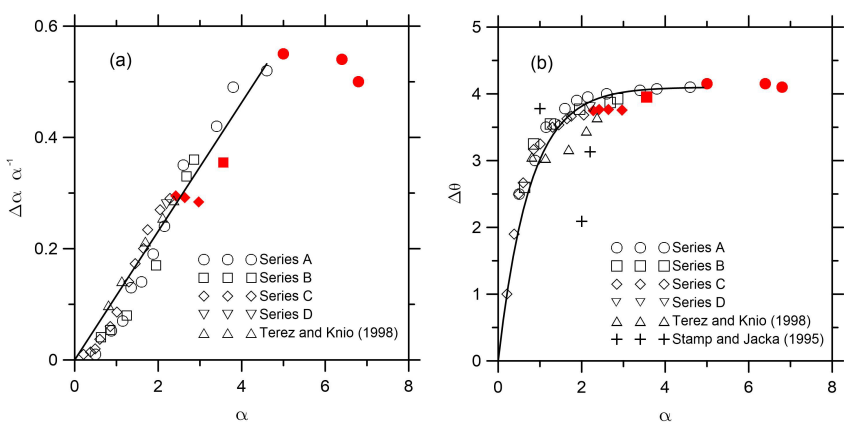

Figure 4. Relative runup excess $\Delta \alpha$ (a) and phase shift $\Delta \theta$ (b) of the interacting symmetric ISWs versus the normalized amplitude of the wave $\alpha$. The filled symbols correspond to the cases with KH instability. The fits, done by using a straight line in panel (a) and an exponential function in panel (b), are shown.

\subsection{Interaction of internal waves with stable trapped cores}

The large-amplitude ISWs with $1.2 \lesssim F r_{\max } \lesssim 1.3$ and $0.15 \lesssim R i_{\min }<1$ are characterized by stable long-lived cores. Figure 6a shows the collision of waves with equal amplitude $\alpha=\alpha_{\mathrm{L}}=\alpha_{\mathrm{R}}=3.3$ (case (A9; A9)) with the parameters $F r_{\max }=1.28$ and $R i_{\min }=0.25$. As seen in the figure, the volumes of dyed fluid in the trapped core collide together with the waves. The cores did not mix during the collision, which was also observed in a laboratory experiment (Honji and Matsunaga, 1995). Then, the outgoing waves captured the cores and carried the dyed fluid in the opposite directions with little mass loss. Some mass exchange between waves 

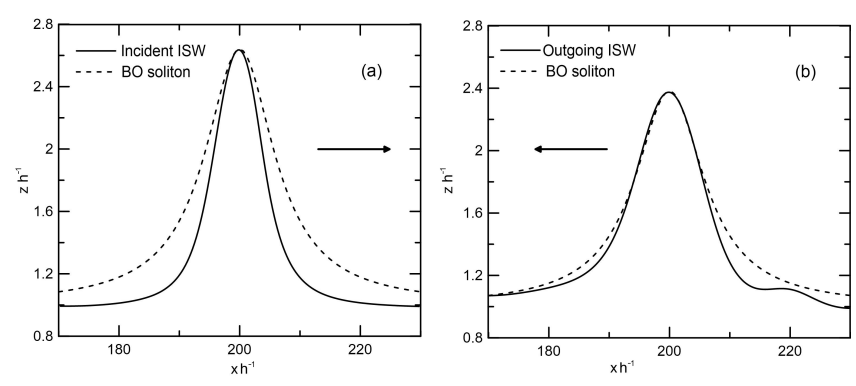

Figure 5. Wave profile of a wave before (a) and after (b) collision in section $x_{\mathrm{L}}$ (b) for $\alpha=1.6$ (case A5; A5). These profiles are compared with the profile of the BO soliton.

that occurred in the mode-2 experiment (Stamp and Jacka, 1995) was, perhaps, the result of a slight displacement of the pycnocline in the vertical direction, which is often observed in laboratory experiments (Carr et al., 2015).

The interaction process is described in Fig. 7 in more detail. Here, the velocity and vorticity fields are shown together with an isopycnal distribution. At the beginning of collision (Fig. 7a), the trapped cores almost touch. They form a pair of vortices carrying trapped fluid upward. The next snapshot (Fig. 7b) corresponds to the time when the potential energy of the interacting waves reaches a maximum and the kinetic energy reaches a minimum. Unlike waves of class (i), at this moment in time, the kinetic energy of the waves does not vanish because the flows in the trapped cores change sign due to the collision. This process is also different from the process of the formation of waves with captured cores due to the collapse of the mixed region, which was initially in a state of rest. Then, the fluid in the cores is entrained by the outgoing waves with some mixing, as represented in a 2-D context, arising due to instability. This results in the slight loss of mass from the trapped cores and a decrease in the phase velocity of $8 \%$ (Figs. 7c and 6a). As shown in Fig. 4a, for stable waves of class (ii), the runup excess $\Delta \alpha / \alpha$ still almost linearly increases in the range $2.3 \leq \alpha \leq 4.6$, whereas the increase in the phase shift $\Delta \theta$ is substantially slowed down when $\alpha>1$, and then $\Delta \theta$ tends towards a constant value at $\alpha \geq 4$. The distributions of $\Delta \alpha$ and $\Delta \theta$ in Fig. 4 for stable waves showed approximated linear and exponential dependencies, respectively, which were based on the weakly nonlinear asymptotics $\Delta \alpha / \alpha \sim \alpha$ and $\Delta \theta \sim \alpha$ (Matsuno, 1998) for small $\alpha$, and obtained in numerical experiments almost constant distribution of $\Delta \theta$ at large $\alpha$. These fitted curves are

$\Delta \alpha=0.116 \alpha, \quad \Delta \theta=4.1[1-\exp (-1.33 \alpha)]$.

The behaviour of mode-2 ISWs during reflection off a solid vertical wall is similar to that of the collision of two waves of equal amplitude. A comparison with the simulated reflection of ISWs off a vertical wall (case D1) in a laboratory experiment (Stamp and Jacka, 1995) is given in Fig. 8. The parameters of the experiment were as follows: density differ- ence $\Delta \rho / \rho_{0}=0.05$, pycnocline thickness $h=0.0025 \mathrm{~m}$, and $\alpha=2.2$. The incident wave belongs to the class (ii) of ISWs: $F r_{\max }=1.18, R i_{\min }=1.05$. The calculated density isopycnals in a vertical cross section along the flume at $t=16 \mathrm{~s}$ in Fig. 8a agree with the density isopycnals visualized in the experiment by water-insoluble droplets of different densities in Fig. 8b. The interaction process is similar to that shown in Fig. 6a, where after collision, some instability and mixing are observed in the rear of the trapped core. In simulated case D1, the corresponding values of $F r_{\max }$ and $R i_{\min }$ after reflection are 1.1 and 1.21, respectively. The simulated and observed trajectories of the wave crests, as shown in Fig. 8c, are similar. The corresponding simulated runup excess was $\Delta \alpha=0.28$ and phase shift $\Delta \theta=3.8$. These values agree with the other values of $\Delta \alpha$ and $\Delta \theta$ in Fig. 4. The experimentally observed (Stamp and Jacka, 1995) phase shift values are also given in Fig. 4b. They demonstrate large scatter due to difficulties encountered in the experiment, as indicated by Stamp and Jacka (1995).

\subsection{Interaction of internal waves with unstable trapped cores}

The large-amplitude ISWs with $F r_{\max } \approx 1.3$ and $R i_{\min } \lesssim 0.1$ belong to class (iii), which is characterized by a local waveinduced shear instability resulting in the appearance of the Kelvin-Helmholtz (KH) billows (Maderich et al., 2015); globally, however, this wave-/self-generated shear system can be stable, as noted by (Almgren et al., 2012). The waves carry out trapped fluid, but the cores gradually lose trapped fluid to the wake through $\mathrm{KH}$ billows shifting to the wave rear and through recirculation in the trapped core (Terez and Knio, 1998; Maderich et al., 2001; Lamb, 2002). Figure $6 \mathrm{~b}$ shows the collision of waves with equal amplitude $\alpha=\alpha_{\mathrm{L}}=\alpha_{\mathrm{R}}=6.4$ (case (A13; A13), with the parameters $F r_{\max }=1.31$ and $R i_{\min }=0.06$ ). Unlike that shown in Fig. $6 \mathrm{a}$ (case (A9; A9)), the collision of trapped cores was accompanied by billows, resulting in mixing. The divergent waves remained locally unstable, again forming $\mathrm{KH}$ billows in the wave aft. The amplitudes of diverging waves gradually decreased due to the loss of mass of the trapped cores.

In the ocean and in most of the laboratory experiments the Schmidt number is about $700-800$. The used grid does not allow the whole range of inhomogeneities in salinity (density) to be resolved. Therefore, it is important to evaluate the effect of molecular diffusion of salinity on the dynamics of waves and to verify the possibility that diffusion can be neglected in the wave collision for large $S c$. Two cases for large-amplitude waves were considered: (A9; A9) and (A13; A13). We performed runs for $S c=1,10$, and 1000. In the collision case (A9; A9) the behaviour of colliding waves is the same, whereas the difference between runs for $S c=1$ and $S c=1000$ was less than $1 \%$ of $\Delta \alpha / \alpha$ and $\Delta \theta$ values. The comparison of the density snapshots during collision in case (A13; A13) for different Schmidt numbers is shown in 
(a)

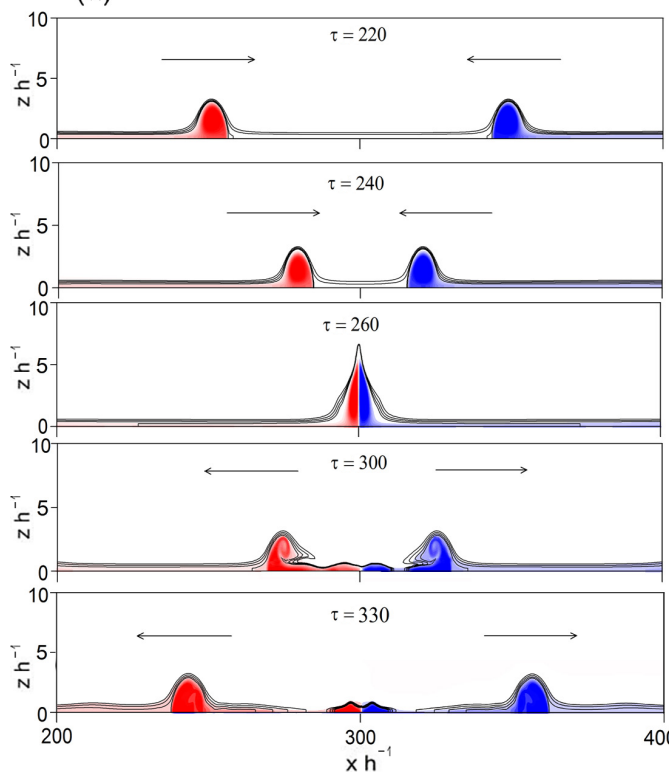

(b)

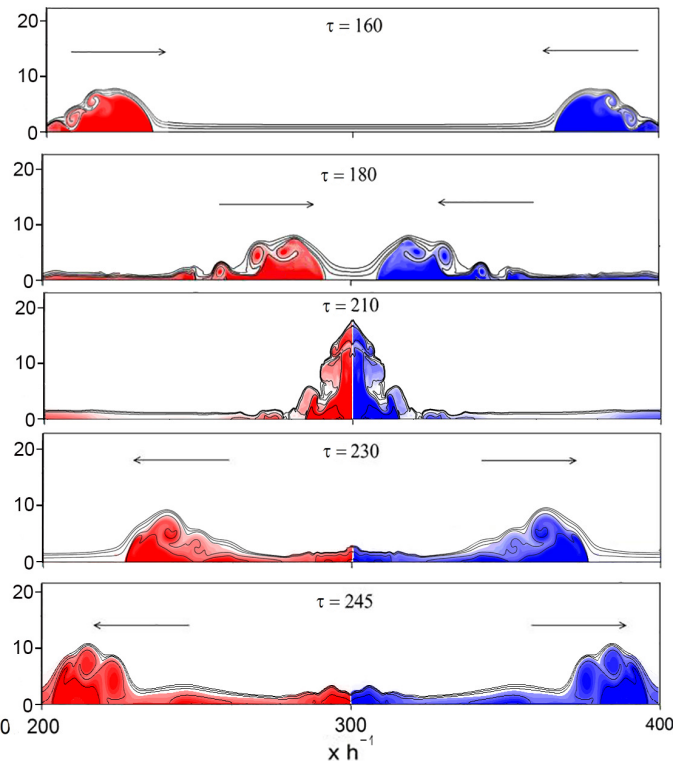

Figure 6. Snapshots of the density isopycnals during the collision of ISWs. (a) Case (A9; A9). (b) Case (A13; A13). The trapped cores are visualized by dyed fluid.

(a)

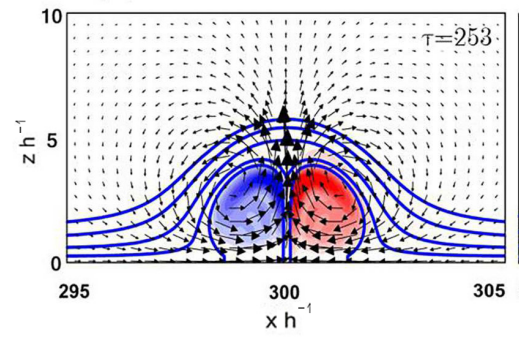

(b)

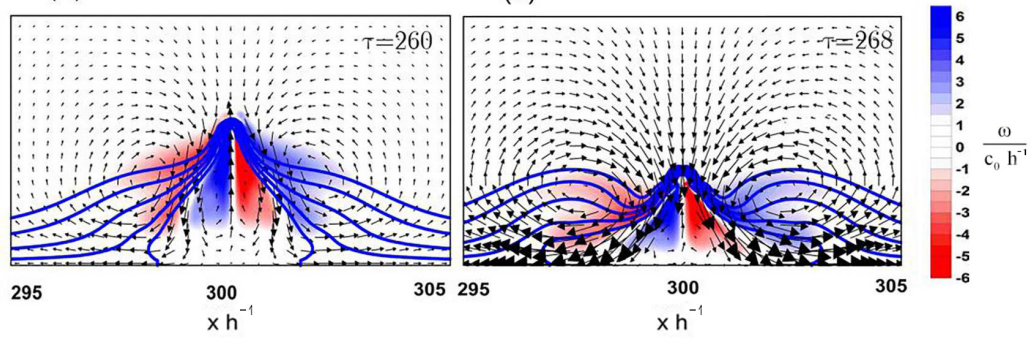

Figure 7. Details of the interaction of waves of equal amplitude $\alpha=3.3$ at different times (case (A9; A9)) in a 2-D setting. The velocity, vorticity $\omega$, and isopycnals are shown.

Fig. 9. Figure 9 clearly depicts the difference between the structure of interacting waves for cases $S c=1$ and $S c=10$. The corresponding values of $\Delta \alpha / \alpha$ and $\Delta \theta$ differ by 5 and $0.6 \%$, respectively. This was in agreement with the results of Deepwell and Stastna (2016), where the essential effect of molecular diffusivity on the mass transport by mode-2 ISW in the range $1 \leq S c<20$ was shown. At the same time, the results of calculations at $S c=10$ and $S c=1000$ in Fig. 9b and c practically coincide, which indicates that molecular diffusion may not be taken into account when studying the global properties of colliding waves. This conclusion agrees with Terez and Knio (1998), as they estimate that the value of $S c=100$ was "sufficiently high for density diffusion to be ignored during the simulation period", and the results of the Deepwell and Stastna (2016) simulation, according to which the mass transfer is virtually independent of $S c$ already at $S c>20$. However, diffusion can be important for small-scale mixing processes in tiny density structures (see e.g. Galak- tionov et al., 2001) forming as a result of instability and turbulent cascade processes (Deepwell and Stastna, 2016) and persisting over time in a wake behind a moving bulge of trapped fluid (Terez and Knio, 1998). These subgrid-scale structures in our simulations were smeared by numerical diffusion which did not affect the larger scale due to use of a second-order total variation diminishing (TVD) scheme for advective terms in the transport equation.

\subsection{Interaction of internal waves with trapped cores and different amplitudes}

The collision process is modified for waves of different amplitudes by the exchange of trapped fluid between colliding waves due to the conservation of momentum (Stamp and Jacka, 1995). This process is shown in Fig. 10 for two cases. In the first case, two stable strongly non-linear waves with trapped cores collide $\left(\alpha_{\mathrm{L}}=3.3\right.$ with $F r_{\max }=1.28, R i_{\min }=$ 


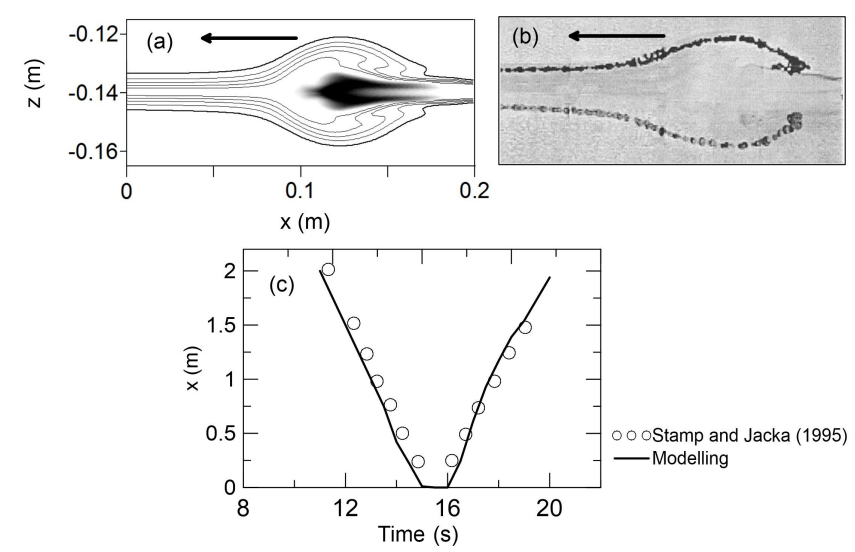

Figure 8. Comparison of the simulated reflection of ISWs off a vertical wall (case D1) in a 2-D setting with a laboratory experiment (Stamp and Jacka, 1995). (a) Snapshot of the calculated density isopycnals, visualized by a black tracer trapped fluid at $t=16 \mathrm{~s}$. (b) Density isopycnals in the experiment, visualized by water-insoluble droplets of different densities. (c) Spatio-temporal diagrams of the path of an ISW during reflection off a wall.

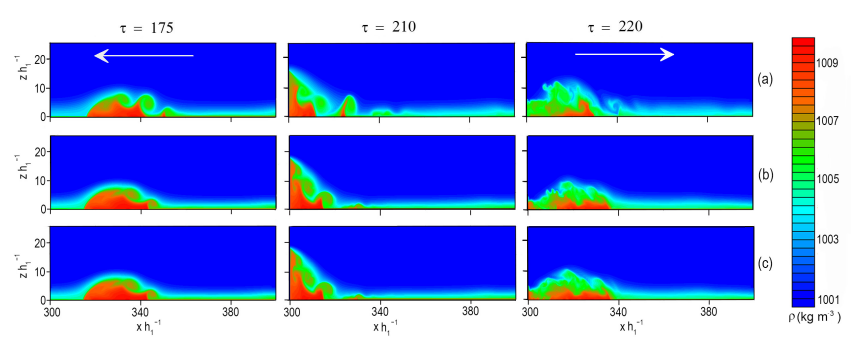

Figure 9. Comparison of the density snapshots during collision of ISWs in case (A13; A13) for different Schmidt numbers. (a) $S c=1$. (b) $S c=10$. (c) $S c=1000$. The right half of the numerical flume is shown due to the symmetry of the interaction process.

0.25; $\alpha_{\mathrm{R}}=2.15$ with $\left.F r_{\max }=1.16, R i_{\text {min }}=0.4\right)$. As seen in Fig. 10a, the part of the trapped core fluid from the wave of larger amplitude (blue colour) is merged with the trapped fluid from the smaller wave (red colour) without noticeable mixing. The circulation inside the core of the larger outgoing wave results in the stirring of the fluid in such a way that the smaller core fluid eventually ends up inside the fluid from the larger wave.

The collision of an ISW of small amplitude (class (i)) with a stable wave of large amplitude (class (ii)) was considered for case (A11; A1) to study the possibility of triggering instability in the wave of large amplitude via a small disturbance, similar to the waves of mode- 1 in a two-layer fluid (Almgren et al., 2012). The simulation results are shown in Fig. 10b. As seen in the figure, the small-amplitude ISW ( $\alpha=0.5$, $\left.F r_{\text {max }}=0.33, R i_{\text {min }}=81\right)$ triggered instability in the ISW with an amplitude that was 10 times larger than that of the small wave. Note that the large-amplitude wave has parameters $\left(\alpha=4.6, F r_{\max }=1.3, R i_{\min }=0.15\right)$ that are close to critical for the development of instability. The amplitude of the small wave essentially decreased during the interaction process due to the interaction and due to the viscous attenuation at the low Reynolds number of the wave $\left(R e_{\mathrm{m}}=45.1\right)$. Unlike the head-on collision of large-amplitude mode- 1 and weakly non-linear mode-2 ISWs (Stastna et al., 2015), the outgoing wave of small amplitude did not degenerate. Spatiotemporal diagrams for the paths of two ISWs of different amplitudes colliding head-on are shown in Fig. 11 for cases (A9; A7) and (A1; A11). As seen in Fig. 11a, the trajectories of waves of larger amplitude propagating from left to right were less subject to changes due to the collision, whereas the phase shift and the decrease in phase velocity for the smaller waves propagating from right to left were essentially greater.

\subsection{Estimation of the energy loss due to collision}

We defined the relative energy loss due to the wave collision $\left(\Delta E_{\text {loss }}\right)$ as the difference between the total loss of energy $E_{\text {tot }}$ due to the collision and the loss of energy by two single waves due to the viscous decay or instability $\Delta E_{\text {dis }}$ :

$\Delta E_{\mathrm{loss}}=\Delta E_{\mathrm{tot}}-\Delta E_{\mathrm{dis}}$.

The relative loss of energy due to the collision of ISWs can be calculated as the normalized difference in energy of waves before and after collision,

$$
\begin{aligned}
\Delta E_{\mathrm{tot}} & =\frac{\mathrm{PSE}_{\mathrm{L}}^{(\mathrm{bf})}+\mathrm{PSE}_{\mathrm{R}}^{(\mathrm{bf})}-\mathrm{PSE}_{\mathrm{L}}^{(\mathrm{af})}-\mathrm{PSE}_{\mathrm{R}}^{(\mathrm{af})}}{\mathrm{PSE}_{\mathrm{L}}^{(\mathrm{bf})}+\mathrm{PSE}_{\mathrm{R}}^{(\mathrm{bf})}}, \\
\Delta E_{\mathrm{dis}} & =\frac{\mathrm{PSE}_{\mathrm{L}}^{(\mathrm{bf})}+\mathrm{PSE}_{\mathrm{R}}^{(\mathrm{bf})}-\widetilde{\mathrm{PSE}}_{\mathrm{L}}^{(\mathrm{af})}-\widetilde{\mathrm{PSE}}_{\mathrm{R}}^{(\mathrm{af})}}{\mathrm{PSE}_{\mathrm{L}}^{(\mathrm{bf})}+\mathrm{PSE}_{\mathrm{R}}^{(\mathrm{bf})}},
\end{aligned}
$$

where $\operatorname{PSE}_{\mathrm{L}}^{(\mathrm{bf})}$ and $\mathrm{PSE}_{\mathrm{R}}^{(\mathrm{bf})}$ are the pseudo-energies of the waves before collision at cross sections $x_{\mathrm{L}}$ and $x_{\mathrm{R}}$, respectively, and $\mathrm{PSE}_{\mathrm{L}}^{(\mathrm{af})}$ and $\mathrm{PSE}_{\mathrm{R}}^{(\mathrm{af})}$ are the pseudo-energies of the waves after collision at cross sections $x_{\mathrm{L}}$ and $x_{\mathrm{R}}$, respectively, whereas $\widetilde{P S E}_{\mathrm{L}}^{\text {(af) }}$ and $\widetilde{P S E}_{\mathrm{L}}^{\text {(af) }}$ are the energies of the outgoing waves without interaction at cross sections $x_{\mathrm{L}}$ and $x_{\mathrm{R}}$, respectively. The pseudo-energy is the sum of the kinetic and available potential energies (Shepherd, 1993) of waves before and after collision. The method for estimation of the available potential energy and energy fluxes was given in Scotti et al. (2006) and Lamb (2007). A detailed description of the procedure of the pseudo-energy calculation was presented by Maderich et al. (2010).

From dimensional arguments, $\Delta E_{\text {loss }}=\Phi\left(\alpha, R e_{\mathrm{m}}, S c\right)$, where $\Phi$ is a function of three arguments. Assuming complete similarity on the $R e_{\mathrm{m}} \rightarrow \infty$ and $S c \rightarrow \infty$, consider dependence $\Delta E_{\text {loss }}$ on $\alpha$. As seen in Fig. 12, this dependence given for symmetric collisions $\left(\alpha=\alpha_{\mathrm{L}}=\alpha_{\mathrm{R}}\right)$ is not monotonic and is not universal, changing depending on the series of calculations. It can be divided into three different ranges. In range $\mathrm{I}(0 \leq \alpha \leq 1)$, the energy loss due to the interaction increases as the wave amplitude increases. This range 
(a)
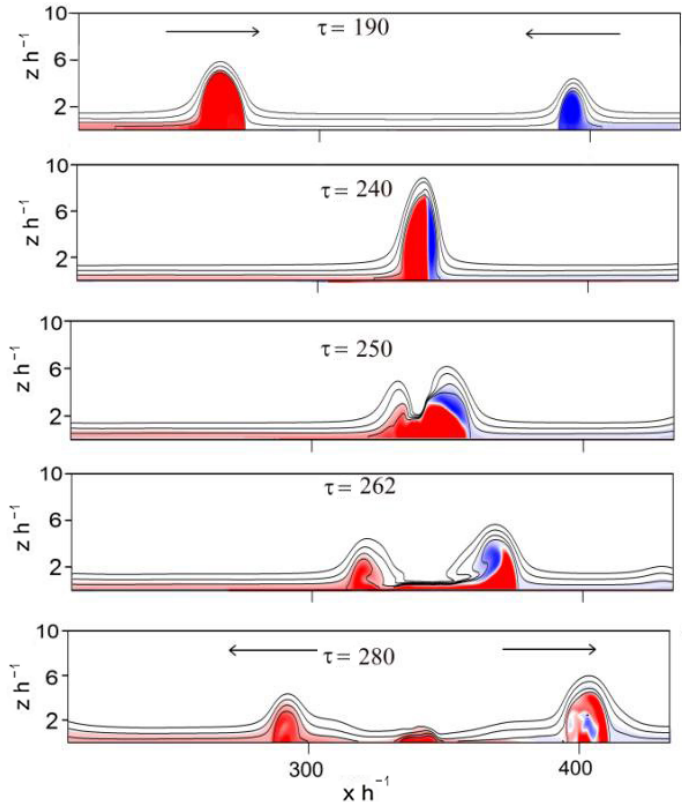

(b)
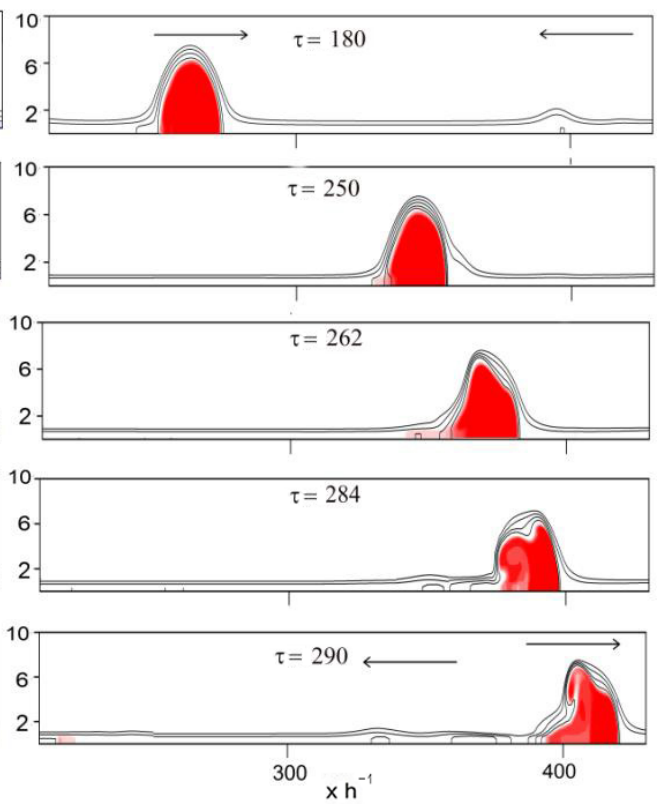

Figure 10. Snapshots of the density isopycnals during the collision of ISWs with different amplitudes for case (A9; A7) (a) and case (A11; A1) (b). The trapped cores are visualized by dye.
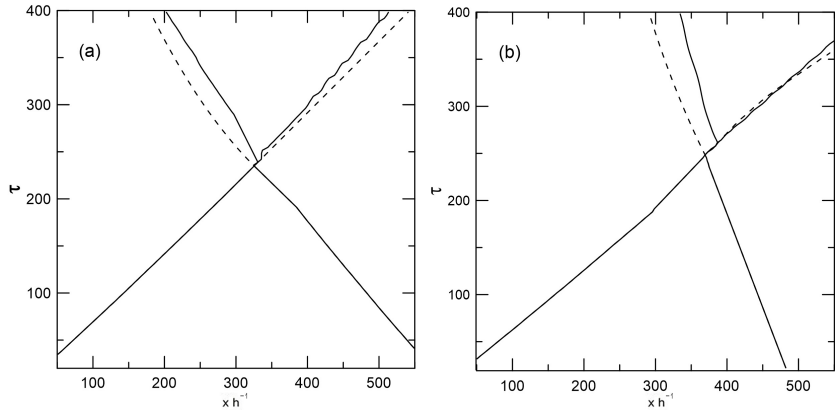

Figure 11. Spatio-temporal diagrams for paths of two ISWs of different amplitudes colliding head-on. (a) Case (A9; A7). (b) Case (A11; A1). The diagrams for the waves without interaction are shown by dashed lines.

coincides with the range of weakly non-linear waves without trapped cores. In range II $(1<\alpha \leq 1.75)$, the relative energy losses reach a maximum. The range coincides with the range in which colliding waves lose trapped cores in the process of interaction. This fact can explain the relative maximum of energy loss as the loss of potential energy of the cores. In range III $(1.75 \leq \alpha)$, the behaviour of the loss of energy is also non-monotonic and non-similar. At first, in the zone of stable large-amplitude collisions, the loss of energy decreases, but as the amplitudes of collided waves increase, the interaction is accompanied by the development of instability; therefore, the loss of energy increases. Finally, for unsta-

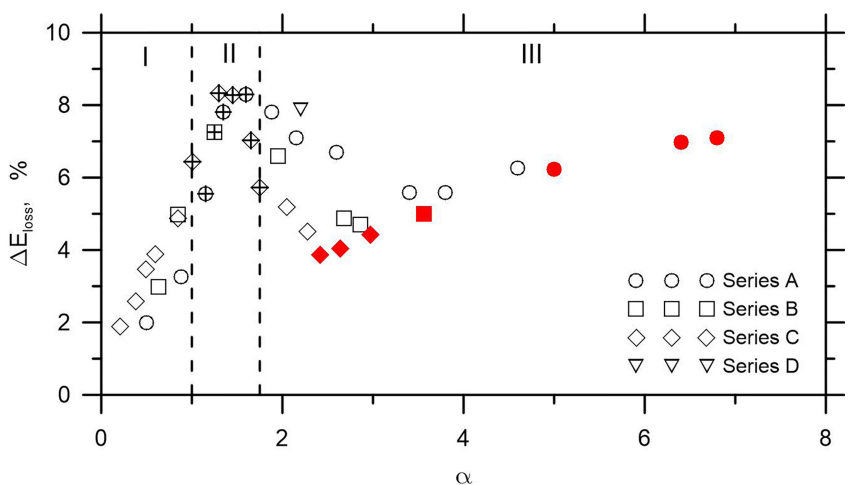

Figure 12. Plot of the energy loss versus the amplitude of the equal colliding waves. The filled symbols correspond to the cases with $\mathrm{KH}$ instability. The crossed symbols correspond to the cases where colliding waves lost trapped cores in the process of interaction.

ble waves, the energy losses due to the interaction increase monotonically with increasing amplitude.

The absence of complete self-similarity in the Reynolds and Schmidt numbers also means that the Euler equations do not describe the wave interaction processes in deep water even for the range of stable waves. As shown in Table 1, the parameter $R e_{\mathrm{m}}$ varies in Series A-C several times for waves of the same dimensionless amplitude $\alpha$. The incomplete similarity scaling following (Barenblatt, 1996) results in the relation $\Delta E_{\text {loss }} \sim \Psi(\alpha) R e_{\mathrm{m}}^{m} S c^{n}$, where $\Psi$ is a function, and $m$ and $n$ are exponents. However, this rescaling also 
did not result in universal dependence. We conclude that this is due to the different mechanisms governing collision processes in ranges I-III: non-linear wave interaction, collapse of collided trapped masses, and instability. Another factor influencing the interaction may be the diffusivity effect (Deepwell and Stastna, 2016), which is described by the Schmidt number. However, in these experiments, the Schmidt number was large and constant.

\section{Conclusions}

The dynamics and energetics of a head-on collision of internal solitary waves (ISWs) with trapped cores propagating in a thin pycnocline were studied numerically within the framework of the Navier-Stokes equations for a stratified fluid. The peculiarity of this collision is that it involves the trapped masses of a fluid. The interaction of ISWs was different for three classes of waves: (i) weakly non-linear waves without trapped cores, (ii) stable strongly non-linear waves with trapped cores, and (iii) shear unstable strongly nonlinear waves with trapped cores. The simulations showed that the wave phase shift grew as the amplitudes of the interacting waves increased for interacting waves of classes (i) and (ii) and remained almost constant for those of class (iii). The excess of the maximum runup amplitude over the sum of the amplitudes of colliding waves increased almost linearly as the amplitude of the interacting waves belonging to classes (i) and (ii) increased. However, it decreased somewhat for those of the unstable class (iii). The dependence is similar to the interaction of the mode- 1 waves in a two-layer stratification (Terletska et al., 2017), with the difference being that the phase shift continues to grow for the collision of interfacial waves of mode-1. The waves of class (ii) with a normalized thickness of the pycnocline amplitude $\alpha$ fully lost fluid trapped by the wave cores in the approximate range of $1 \leq \alpha \leq 1.75$. The interacting stable waves of higher amplitude captured cores and carried trapped fluid in the opposite directions with little mass loss. The collision of locally shear unstable waves of class (iii) was accompanied by the development of instability. We concluded that this kind of interaction reduced the capacity of an ISW to transport mass. The dependence of energy loss on wave amplitude was not monotonic. Initially, the energy loss due to the interaction increased with increasing wave amplitude. Then, the energy losses reached a maximum due to the loss of potential energy of the cores upon collision, and then started to decrease. With further amplitude growth, the collision was accompanied by the development of instability, and the loss of energy increased. The collision process was modified for waves of different amplitudes because of the exchange of trapped fluid between colliding waves due to the conservation of momentum. Merging of the trapped fluid due to the collision of stable waves belonging to class (ii) occurred through the stirring mechanism without noticeable mixing. Similar to waves of mode-1 in a two-layer fluid (Almgren et al., 2012; Terletska et al., 2017), the interaction of a wave of large amplitude with a wave of small amplitude can trigger local wave instability of the large-amplitude wave if the parameters of this wave were close to critical for the development of instability. The results obtained can be applied to the interaction dynamics of a subsurface trapped core formed within a shoaling largeamplitude internal wave (Lien et al., 2012). Note, however, that the destruction of the KH billows is essentially a 3-D process; therefore, 3-D high-resolution simulation is necessary to predict turbulence development (Arthur and Fringer, 2014; Deepwell and Stastna, 2016). This is the subject of a separate study, whereas the interaction of the colliding waves as a whole can be described in a 2-D setting.

Data availability. No data sets were used in this article.

Competing interests. The authors declare that they have no conflict of interest.

Special issue statement. This article is part of the special issue "Extreme internal wave events". It is a result of the EGU, Vienna, Austria, 23-28 April 2017.

Acknowledgements. This work was supported by funds from CKJORC and the major research project of KIOST (PE99501). The authors are grateful to Oliver Fringer, an anonymous referee, and handling editor Marek Stastna for their inspiring and constructive comments, which were very helpful in improving the manuscript.

Edited by: Marek Stastna

Reviewed by: Oliver Fringer and one anonymous referee

\section{References}

Almgren, A., Camassa, R., and Tiron, R.: Shear instability of internal solitary waves in Euler fluids with thin pycnoclines, J. Fluid Mech., 710, 324-361, 2012.

Arthur, R. S. and Fringer, O. B.: The dynamics of breaking internal solitary waves on slopes, J. Fluid Mech., 761, 360-398, 2014.

Barenblatt, G. I.: Scaling, self-similarity, and intermediate asymptotics, Cambridge University Press, Cambridge, UK, 1996.

Benjamin, T. B.: Internal waves of permanent form in fluids of great depth, J. Fluid Mech., 29, 559-592, 1967.

Brandt, A. and Shipley, K. R.: Laboratory experiments on mass transport by large amplitude mode-2 internal solitary waves, Phys. Fluids, 26, 046601, https://doi.org/10.1063/1.4869101, 2014.

Carr, M., Fructus, D., Grue, J., Jensen, A., and Davies, P. A.: Convectively-induced shear instability in large internal solitary waves, Phys. Fluids, 20, 126601, https://doi.org/10.1063/1.3030947, 2008. 
Carr, M., Davies, P. A., and Hoebers, R. P.: Experiments on the structure and stability of mode-2 internal solitary-like waves propagating on an offset pycnocline, Phys. Fluids, 27 046602, https://doi.org/10.1063/1.4916881, 2015.

Christie, D. R.: The morning glory of the Gulf of Carpentaria: a paradigm for non-linear waves in the lower atmosphere, Aust. Meteorol. Mag., 41, 21-60, 1992.

Davis, R. E. and Acrivos, A.: Solitary internal waves in deep water, J. Fluid Mech., 29, 593-607, 1967.

Deepwell, D. and Stastna, M.: Mass transport by mode2 internal solitary-like waves, Phys. Fluids, 28, 056606, https://doi.org/10.1063/1.4948544, 2016.

Galaktionov, O., Maderich, V., and Nikishov, V.: Evolution of layered structures in the final stage of turbulent decay in a stably stratified fluid, Dynam. Atmos. Oceans, 34 125-144, 2001.

Gavrilov, N., Liapidevskii, V., and Gavrilova, K.: Mass and momentum transfer by solitary internal waves in a shelf zone, Nonlin. Processes Geophys., 19, 265-272, https://doi.org/10.5194/npg19-265-2012, 2012.

Gear, J. A. and Grimshaw, R.: Weak and strong interactions between internal solitary waves, Stud. Appl. Math., 70, 235-258, 1984.

Grue, J., Jensen, A., Rusas, P.-O. J., and Sveen, K.: Breaking and broadening of internal solitary waves, J. Fluid Mech., 413, 181217,2000

Helfrich, K. R. and Melville, W. K.: Long nonlinear internal waves, Annu. Rev. Fluid Mech., 38, 395-425, 2006.

Helfrich, K. R. and White, B. L.: A model for large-amplitude internal solitary waves with trapped cores, Nonlin. Processes Geophys., 17, 303-318, https://doi.org/10.5194/npg-17-303-2010, 2010.

Honji, H. and Matsunaga, N.: Sugihara Y and Sakai K Experimental observation of internal symmetric solitary waves in a two-layer fluid, Fluid Dyn. Res., 15, 89-102, 1995.

Kanarska, Y. and Maderich, V.: A non-hydrostatic numerical model for calculating free-surface stratified flows, Ocean Dynam., 53, 176-185, 2003.

Kao, T. W. and Pao, H. P.: Wake collapse in the thermocline and internal solitary waves, J. Fluid Mech., 97, 116-127, 1980.

Klymak, J. M. and Moum, J. N.: Internal solitary waves of elevation advancing on a shoaling shelf, Geophys. Res. Lett. 30, 2045, https://doi.org/10.1029/2003GL017706, 2003.

Lamb, K.: A numerical investigation of solitary internal waves with trapped cores formed via shoaling, J. Fluid Mech., 451, 109-144, 2002.

Lamb, K.: Energy and pseudoenergy flux in the internal wave field generated by tidal flow over topography, Cont. Shelf Res., 27, 1208-1232, 2007.

Lamb, K. and Farmer, D.: Instabilities in an internal solitary-like wave on the Oregon shelf, J. Phys. Oceanogr., 41, 67-87, 2011.

Lien, R. C., D’Asaro, E. A., Henyey, F., Chang, M., Tang, T., and Yang, Y.: Trapped core formation within a shoaling nonlinear internal wave, J. Phys. Oceanogr., 42, 511-525, 2012.

Luzzatto-Fegiz, P. and Helfrich, K.: Laboratory experiments and simulations for solitary waves with trapped cores, J. Fluid Mech., 757, 354-380, 2014.

Maderich, V., van Heijst, G. J., and Brandt, A.: Laboratory experiments on intrusive flows and internal waves in a pycnocline, $\mathrm{J}$. Fluid Mech., 432, 285-311, 2001.
Maderich, V., Talipova, T., Grimshaw, R., Terletska, K., Brovchenko, I., Pelinovsky, E., and Choi, B. H.: Interaction of a large amplitude interfacial solitary wave of depression with a bottom step, Phys. Fluids, 22, 076602, https://doi.org/10.1063/1.3455984, 2010.

Maderich, V., Brovchenko, I. Terletska, K., and Hutter, K.: Numerical simulations of the nonhydrostatic transformation of basinscale internal gravity waves and wave-enhanced meromixis in lakes, chap. 4, in: Nonlinear internal waves in lakes, edited by: Hutter, K., Series: Advances in Geophysical and Environmental Mechanics, Springer, Heidelberg, Germany, 2012.

Maderich, V., Jung, K. T., Terletska, K., Brovchenko, I., and Talipova, T.: Incomplete similarity of internal solitary waves with trapped core, Fluid Dyn. Res., 47, 035511, https://doi.org/10.1088/0169-5983/47/3/035511, 2015.

Matsuno, Y.: Oblique interaction of interfacial solitary waves in a two-layer deep fluid, P. Roy. Soc. Lond. A, 454, 835-856, 1998.

Maxworthy, T.: On the formation of nonlinear internal waves from the gravitational collapse of mixed regions in two and three dimensions, J. Fluid Mech., 96, 47-64, 1980.

Mellor, G. L.: An equation of state for numerical models of ocean and estuaries, J. Atmos. Ocean. Tech., 8, 609-611, 1991.

Moum, J. N., Farmer, D. M., Smyth, W. D., Armi, L., and Vagle, S.: Structure and generation of turbulence at interfaces strained by internal solitary waves propagating shoreward over the continental shelf, J. Phys. Oceanogr., 33, 2093-112, 2003.

Ono, H.: Algebraic solitary wave in stratified fluid, J. Phys. Soc. Jpn., 39, 1082-1091, 1975.

Ramp, S. R., Yang, Y. J., Reeder, D. B., Buijsman, M. C., and Bahr, F. L.: The evolution of mode-2 nonlinear internal waves over the northern Heng-Chun Ridge south of Taiwan, Nonlin. Processes Geophys., 22, 413-431, https://doi.org/10.5194/npg22-413-2015, 2015.

Reeder, M. J., Christie, D. R., Smith, R. K., and Grimshaw, R.: Interacting "morning glories" over northern Australia, B. Am. Meteorol. Soc., 76, 1165-1171, 1995.

Salloum, M., Knio, O. M., and Brandt, A.: Numerical simulation of mass transport in internal solitary waves, Phys. Fluids, 15, 89102, 2012.

Scotti, A. and Pineda, J.: Observation of very large and steep internal waves of elevation near the Massachusetts coast, Geophys. Res. Lett., 31, L22307, https://doi.org/10.1029/2004GL021052, 2004.

Scotti, A., Beardsley, R., and Butman, B.: On the integration of energy and energy fluxes of nonlinear internal waves: An example from Massachusetts Bay, J. Fluid Mech., 561, 103-112, 2006.

Shepherd, T. G.: A unified theory of available potential-energy, Atmos. Ocean, 31, 1-26, 1993.

Shroyer, E. L., Moum, J. N., and Nash, J. D.: Mode 2 waves on the continental shelf: Ephemeral components of the nonlinear internal wavefield, J. Geophys. Res., 115, C07001, https://doi.org/10.1029/2009JC005605, 2010.

Stamp, A. P. and Jacka, M.: Deep-water internal solitary waves, J. Fluid Mech., 305, 347-371, 1995.

Stastna, M., Olsthoorn, J., Baglaenko, A., and Coutino, A.: Strong mode-mode interactions in internal solitary-like waves, Phys. Fluids 27, 046604, https://doi.org/10.1063/1.4919115, 2015. 
Terez, E. D. and Knio, O. M.: Numerical simulations of largeamplitude internal solitary waves, J. Fluid Mech., 362, 53-82, 1998.

Terletska, K., Jung, K. T., Maderich, V., and Kim, K. O.: Frontal collision of internal solitary waves of first mode, Wave Motion, https://doi.org/10.1016/j.wavemoti.2017.12.006, in press, 2017.
Yang, Y. J., Fang, Y. C., Tang, T. Y., and Ramp, S. R.: Convex and concave types of second baroclinic mode internal solitary waves, Nonlin. Processes Geophys., 17, 605-614, https://doi.org/10.5194/npg-17-605-2010, 2010. 